\title{
Biomarkers of sepsis in neonates and children
}

BY JOSKO MARKIC

\section{Abstract}

Sepsis, and related complications, is still a common cause of death in hospitalized patients worldwide, especially in critically ill neonates and children. Sepsis is also responsible for significant morbidity, and financial burden. It is very important to recognize sepsis early, since delayed diagnosis is associated with worse outcome. The early detection of sepsis remains a great challenge for clinicians because the use of blood cultures, the gold standard for diagnosis of bacteremia, is fraught with difficulties. The role of different immune and metabolic biomarkers is to improve the diagnosis, treatment and prognosis of sepsis. White blood cell count, C-reactive protein and procalcitonin are currently the most widely used biomarkers, but they have limited abilities to distinguish sepsis from other inflammatory conditions or to predict outcome. In this review, these biomarkers will be discussed along with novel diagnostic, prognostic and treatment response biomarkers, including interleukins $-6,-8,-18$, tumor necrosis factor - alpha, CD11b, CD64 and CD15s. The future of sepsis biomarkers lies in extensive validation studies of all novel biomarkers and their combinations as early predictors of sepsis. Also, research to identify novel sepsis biomarkers and develop specific therapeutic strategies based on biomarker information has to be continued.

Key words: infant, child, biomarkers, CD15s antigen, C-reactive protein, procalcitonin 


\section{Introduction}

Serious bacterial infections (SBI) are among the leading causes of morbidity and mortality in children worldwide, with a reported rate in young febrile infants between $8.5 \%$ and $12 \%$. (1-3) Sepsis, a diagnosis established according to criteria defined by Levy et al. (4), including documented or suspected infection and findings of inflammation such as hemodynamic instability, tissue perfusion alteration and indications of organ dysfunction, is a leading cause of death in critically ill patients despite the use of broad spectrum antibiotics and modern resuscitation therapies. (5) Besides sepsis, the SBI group includes microbiologically confirmed bacteraemia, bacterial meningitis, urinary tract infection, pneumonia confirmed by an infiltrate on chest x ray, bacterial gastroenteritis, or cellulitis. (6) Early recognition of SBI is associated with better treatment outcome. (7) Unfortunately, diagnosis of bacterial infections is sometimes challenging, because the use of blood culture, the gold standard for diagnosis of bacteremia, is fraught with difficulties. First, incubation of bacteria may take several days (2-4 days). Second, genuine bacteremia may remain undetected in a large proportion of infected cases because of the small volume of blood taken. Third, bacteremia in the neonate may often be transient or intermittent, especially during the early stages of infection. (8) All of this has led to the investigation of various other biomarkers.

Biomarkers are extremely important in the process of diagnosis of sepsis and evaluation of its severity because they can indicate the presence or absence or severity of sepsis, (9) and can help differentiate bacterial from viral and/or fungal infection. It remains difficult to differentiate sepsis from other noninfectious causes of systemic inflammatory response syndrome, and there is continuous search for better biomarkers of sepsis. Based on the National Institutes of Health consensus conference in 2001, (10) a biomarker is defined as a characteristic that can be measured objectively and evaluated as an indicator of normal biological processes, pathologic processes, or pharmacologic responses to a therapeutic intervention. There are four major types of biomarkers: diagnostic, monitoring, stratification and surrogate biomarkers. $(9,11)$ Diagnostic 
biomarkers serve to identify the presence or absence of a disease or other clinical condition. The majority of diagnostic biomarkers are of most value within a specific clinical context since it is very rare that a diagnostic biomarker is strictly present or absent. The presence of a biomarker is measured and cut-offs are defined to establish the presence or absence of disease. This class includes the subset of screening biomarkers. Monitoring biomarkers are molecules or proteins whose levels change dynamically as a disease process evolves or in response to applied therapeutic interventions. Valuable monitoring biomarkers must, therefore, be functionally or biologically linked to the mechanism of action of a given therapeutic intervention. Stratification biomarkers are useful to sort a population of patients into different classes of severity. An effective and useful stratification biomarker can identify higher risk patients who would benefit from more toxic, more aggressive, or higher risk therapies. Finally, surrogate biomarkers are used to predict outcome of a disease process rather than follow its course or to titrate therapy. They serve as proxy end points for severe or rare patient-centered outcomes such as death or significant complications.

In order to be clinically useful, a biomarker should have a well-defined cut-off value to differentiate infected from non infected patients, and it should be able to do that at an early stage of disease. For diagnostic purposes, a very high sensitivity and negative predictive value (approaching 100\%) and good specificity and positive predictive value (>85\%) are desirable. (8) However, if the diagnostic marker is unable to satisfy the above criteria, then the optimal cut-off could be chosen so that both the sensitivity and the specificity approach $80 \%$. Second, the kinetics of a biomarker should be considered because a rapid increase of its value would allow a good discrimination between health and disease. Third, with reference to the laboratory characteristics, the infection biomarker should be biochemically stable because it might be necessary to keep blood samples stored.

A set of different tests including the white blood cell (WBC) count, leukocyte differential counting for evidence of a myeloid left shift, erythrocyte sedimentation rate (ESR), C-reactive protein (CRP), procalcitonin (PCT), and microbiologic cultures is used in the laboratory 
evaluation of patients with suspected infection or sepsis. Also, many different clinical scores have been developed to detect patients at high or low risk of sepsis or other SBI. However, none of the evaluated clinical scales and laboratory markers have been shown to be sensitive or specific enough to be used as a tool to determine the absence or presence of SBI in neonates and children with absolutely no doubt. Therefore, the new generation of biomarkers and improved diagnostics for infection have been directed towards soluble biomarkers in the serum or plasma, and towards analysis of leukocyte cell surface molecules. $(12,13)$

White blood cells (WBC) count is the most commonly used biomarker in clinical practice and diagnostic algorithms commonly used to evaluate neonates, infants or children with fever include the interpretation of WBC. Among febrile children a WBC of $<5$ or $\geq 15 \mathrm{x}$ $10^{9} / 1$ is considered to be associated with an increased risk of SBI. However, some studies have indicated that WBC is not a reliable nor accurate indicator of SBI in febrile infants. In 2001, Galletto-Lacour et al. (14) confirmed that total and differential leukocyte count performed poorly compared to PCT and CRP. WBC cut-off of $15 \times 10^{9} / 1$ had a sensitivity of $68 \%$ and specificity of $77 \%$. Band count $>1.5 \times 10^{9} / 1$ sensitivity was $29 \%$, and specificity $91 \%$. (14) Bonsu et al. (15) found that use of the WBC cut-off of $15 \times 10^{9} / 1$ would have yielded a sensitivity of only $45 \%$ and specificity of $78 \%$. Even the use of a lower cut-off of $5 x$ $10^{9} / 1$ would not detect $21 \%$ patients with bacteremia. Similar results were presented by the study done by Galletto-Lacour in 2003. (16) They showed that WBC cut-off of $15 \times 10^{9} / 1$ had a poor sensitivity of $52 \%$ and specificity of $74 \%$. The value of band count was even worse and extremely poor, with sensitivity of only $11 \%$ (16). Rudinsky et al. (17) also found that the suggested cut-offs of a WBC count $<5 \times 10^{9} / 1$ and $>15 \times 10^{9} / 1$ were not accurate and reliable predictor of SBI in infants age 3 to 24 months since no statistical difference was detected in the WBC count between the SBI and non-SBI groups.

C reactive protein (CRP) is an acute-phase reactant synthesized by the liver in response to elevated levels of the proinflammatory cytokines (IL-1, IL-6 and TNF- $\alpha$ ). It is produced within 4-6 hours after onset of 
tissue injury or inflammation, and it doubles every 8 hours before peaking around 36 hours. (18) CRP is frequently used as part of the assessment of children with fever of unknown origin, however, its levels become elevated in any disease process with tissue injury, not just infection. CRP is also among the most widely used biomarkers in neonates. (19) Franz et al. considered a CRP value $>10 \mathrm{mg} / \mathrm{L}$, in the presence of one (or more) clinical sign(s) compatible with infection, as a criterion to make a diagnosis of clinical septicemia in neonates. (20) However, due to the time lag in the response of CRP to infection, some clinicians use it in combination with other biomarkers. Pulliam et al. (21) reported the results of a prospective study in 2001 examining the utility of CRP in evaluating febrile children. Seventy-seven children 1 to 36 months of age were enrolled in this study. CRP was found to be a much more useful biomarker than WBC count for this group of patients, with sensitivity of $79 \%$ and specificity of $91 \%$. Galetto-Lacour et al. (14) also evaluated the utility of CRP as a potential biomarker of infection. Children were prospectively enrolled at a pediatric emergency department in a European university hospital. A CRP cutoff of $40 \mathrm{mg} / \mathrm{l}$ resulted in a sensitivity of $89 \%$ and specificity of $75 \%$; more sensitive than Pulliam et al., (21) but also less specific. Similar results were found by Galetto-Lacour two years later in a follow-up study, (16) which showed that CRP levels were superior to WBC counts in detecting SBIs, with reported sensitivity and specificity of $79 \%$. The study by Olaciregui et al., (6) focused on infants aged $\leq 3$ months, also showed that CRP appears to be useful in predicting SBI and recommended that it should be used in combination with other markers for management of febrile patients in this age group. Other authors, (3) studying the same age group, similarly reported that various cut-offs of CRP had a better sensitivity and specificity than WBC cut-offs. Nevertheless, despite the high specificity of CRP, its sensitivity was low.

Procalcitonin (PCT) is a 116-aminoacid peptide and also an acutephase reactant. PCT level begins to rise 4 hours after exposure to bacterial endotoxin, peaks at 6-8 hours, and remains elevated for at least 24 hours. (22) It has been used to diagnose the severity of systemic inflammatory response, to determine the progression of infection to sepsis and septic shock, to assess responsiveness to treatment, and to 
estimate prognosis. (22) Increases in PCT occur before, and more rapidly, than increases in CRP and its peak level is achieved sooner. Additionally, when the patient responds appropriately to therapy, PCT levels decrease to normal much quicker than those of CRP. The most important clinical applicability of PCT as a biomarker may be in determining the initiation (diagnostic biomarker) and duration (monitoring biomarker) of antibiotic therapy. (11) All studies on severe, invasive bacterial infections in children report higher sensitivities and specificities of PCT than for CRP, (23-25) although its value has also been challenged. (22) In 2003, Galetto-Lacour et al. (16) published the results of their prospective research confirming the superiority of PCT. One hundred and ten febrile children aged 7 days to 36 months presenting to a pediatric emergency department were enrolled. A PCT cutoff of $0.5 \mathrm{ng} / \mathrm{ml}$ resulted in a sensitivity of $93 \%$ and specificity of $74 \%$, similar to the author's previous findings. CRP was less sensitive and specific: the proposed optimum cutoff of $40 \mathrm{mg} / \mathrm{L}$ was $52 \%$ sensitive and $74 \%$ specific. The large study on PCT was that of Gendrel et al. (26) The authors, in a group of children hospitalized after admission to the Emergency Department, found that PCT, at the cut-off of $2 \mathrm{ng} / \mathrm{mL}$, had a very high sensitivity (96\%) and specificity (87\%) in detecting invasive bacterial infection and performed much better than CRP at different cut-offs. Similar results were reported by Fernandez Lopez et al. (23) Four hundred and forty-five children were enrolled. Eighty children were diagnosed with "localized" bacterial infections while 150 patients were diagnosed with "invasive" bacterial infections (sepsis, pyelonephritis, meningitis, and lobar pneumonia). The authors found PCT to be the best predictor of bacterial infection. Using a calculated optimum cut-off of $0.53 \mathrm{ng} / \mathrm{ml}$, the authors reported sensitivity of $65.5 \%$, and specificity of $94.3 \%$. CRP (> $27.5 \mathrm{mg} / \mathrm{L})$ was less sensitive (63.5\%) and less specific (84.2\%). When used to differentiate only those children who were diagnosed with invasive bacterial disease, $\mathrm{PCT} \geq 0.59 \mathrm{ng} / \mathrm{ml}$ had a sensitivity of $91.3 \%$ and specificity of $93.5 \%$, much better than the $78 \%$ and $75 \%$ noted with CRP, respectively. In this study, both PCT and CRP were found to perform better when compared to $\mathrm{WBC}$ and $\mathrm{ANC}$ in detecting invasive infections.

\section{Cytokines}


Sepsis is a syndrome characterized by a systemic inflammatory response in which the cytokines produced by immune cells, in response to bacterial products, are important mediators. Increased plasma levels of cytokines, such as tumor necrosis factor-alpha (TNF-alpha), interleukin (IL)-1 and IL-6 have been found in sepsis, and a significant correlation between high levels of these cytokines and poor clinical outcome has been reported. $(27,28)$ The initial cytokinaemia is accompanied by a compensatory response of raised concentrations of circulating anti-inflammatory cytokines (eg, interleukin 10)

Interleukin 6 (IL-6) is an interesting biomarker for clinicians since its levels appear to rise several hours prior to elevation of CRP in the face of infection, and it was found more useful than IL-1 or TNF-alpha in predicting bacteremia in children. (29) Plasma levels of IL-6 were significantly higher in newborns with proven sepsis and clinical sepsis compared to a control group of newborns. (30) These results showed that IL-6 plasma levels have a high diagnostic specificity for neonatal sepsis (92\%), however with a lower sensitivity of 47\%. (30) Galetto-Lacour et al. (14) also found that levels of IL-6 were significantly higher in the group of children with SBI compared to the benign infection group. Gendrel et al. (26) measured IL-6 levels in febrile children. In their study, IL-6 was lower in patients with viral infections. Surprisingly, some of the highest levels of IL-6 were also found in children with viral infections. In the same study, it was found that for the group with SBI, the calculated area under the curve (AUC) for IL-6 was only 0.78, compared with 0.94 for PCT and 0.78 for CRP. Therefore, the authors noted that IL- 6 was the least useful marker. (26) A similar conclusion was reported by Lacour et al. (14) who examined the usefulness of IL-6 in predicting SBI in febrile children during their 2001 study of CRP. They also found that interleukins were not as useful as PCT or CRP, with lower sensitivities and specificities.

Interleukin-8 (IL-8) is a potent neutrophil activating agent whose elevated levels predict organ failure in adults with septic shock, (31) and serve as a valuable biomarker of neonatal sepsis. $(27,32)$ It was found that IL-8 is superior to CRP in detection of neonatal sepsis (32) and that elevated levels of IL-8 can serve as a promising marker of early-onset 
neonatal sepsis. (33) However, Galetto-Lacour et al. (14) challenged these results and reported that the levels of IL-8 were comparable between the group of children with SBI and the benign infection group.

Interleukin-18 (IL-18) is a proinflammatory cytokine produced by activated macrophages that participates in the induction of cell-mediated immunity. IL-18 is also among biomarkers which are gaining more attention recently as a potential predictor of sepsis, but the overall data are currently not as extensive as that for PCT. (11) Increased IL-18 levels are found in many inflammatory conditions, including neonatal infections, and sepsis. (34) Kingsmore et al. reported that IL-18 and seven other serum biomarkers were elevated in preterm infants who developed symptoms of infection. (35) However, Bender et al. determined in their population of neonates that IL-18 had no diagnostic ability. (36) Therefore, the utility of IL-18 as a diagnostic biomarker for sepsis remains to be further evaluated, and much more study, especially in the pediatric population, is needed to clarify the utility of this biomarker in the diagnosis of sepsis.

Tumor necrosis factor-alpha (TNF-alpha) is another biomarker whose plasma levels were found significantly increased in the group of neonates with proven sepsis compared to the control group of patients. In that study, the sensitivity and specificity of TNF-alpha for the diagnosis of neonatal sepsis were $60 \%$ and $84 \%$, respectively. (30) TNF-alpha is also a sensitive marker and has a high sensitivity and negative predictive value for detecting late onset infection in very low birth weight (VLBW) newborns. (37) In contrast, IL-1 $\beta$ and E-selectin had rather low sensitivity for detecting late onset infection within the first 48 hours in VLBW. In the same article it was shown that the use of multiple markers (IL-6, TNF-alpha and CRP) in combination, was associated with higher sensitivity and better negative predictive values. In particular, the use of CRP with either IL-6 or TNF-alpha seemed to be most sensitive for confirming infection. After calculating all possible combinations and permutations for these three tests within the first 48 hours of infection, CRP and IL-6 on day o combined with either TNF-alpha on day 1 or CRP on day 2 provided the best overall sensitivity (98\%) and specificity (91\%) for the diagnosis of late onset infection. (37) 


\section{Leukocyte cell surface molecules}

Leukocyte cell surface molecules mediate many of the interactions between the immune system and antigens. The relatively new approach to rapid and early diagnosis of infection or sepsis is to utilize some of the molecular changes of the innate immune response to detect the presence of infection or sepsis. Neutrophils exist in three states: resting (unstimulated), primed (encounter with an inflammatory or microbialderived product that has lowered the threshold stimulus needed for activation), and activated (undertaking a defined function). Bacterial elimination is dependent on the rapid recruitment of blood neutrophils into sites of infection. Therefore, the effective movement of leukocytes from within the vasculature to the extravascular space is an extremely important physiological process. This process occurs through a cascade of events typically described as attachment (initial tethering), rolling, firm adhesion, and extravasation. Binding of neutrophils to the vascular endothelium is controlled by the sequential activities of two families of adhesion molecules; the selectins and integrins. The selectins promote the initial rolling or tethering of the neutrophils to the endothelium under the force of blood flow, whereas the integrins induce firm adhesion. (38) There are two subfamilies of integrins ( $\beta 1$ and $\beta 2$ ). The $\beta_{2}$ integrins have a common $\beta$-subunit $(\beta 2, \mathrm{CD} 18)$ and distinct $\alpha$-subunits (CD11a, CD11b, CD11c, and CD11d). On the neutrophil surface, L-selectin (CD62L) interacts with specific oligosaccharide moieties on endothelialcell surface glycoproteins, whereas on the endothelium, E-selectin (CD62E) and P-selectin (CD62P) similarly recognize specific neutrophil carbohydrate motifs. Transient rolling helps neutrophils to make contact with inflammatory factors that are expressed on the endothelial surface. This interaction stimulates the neutrophils to rapidly increase the avidity of the $\beta 2$ integrins for the endothelial ligand. The integrin CD11b/CD18 (complement receptor type 3 [CR3], Mac-1, or $\alpha \mathrm{M} \beta 2$ ) is the predominant $\beta 2$ integrin in neutrophils. In resting non-stimulated neutrophils from adults, $95 \%$ of the total cell content of $\mathrm{CD} 11 \mathrm{~b} / \mathrm{CD} 18$ complexes occurs as membrane components of specific granules and secretory vesicles, i.e., in intracellular storage granules, with only $5 \%$ of the complexes expressed on the cell surface. (39) 
The expression of CD11b on neutrophils in sepsis has been reported to be increased and serves as an activation marker of neutrophils, may predict the development of organ failure in patients with septic shock (31) and is indicative of a poor prognosis. (40) The Turunen et al. study shows that determination of CD11b expression may be useful in early identification of ELBW infants with late-onset sepsis. (41) The results of this study demonstrated that CD11b expression has increased in some infants from the infection group up to 3 days before the onset of symptoms suggesting that CD11b measurements may enable, at least in some infants, early diagnosis of infection. It has been also found that an increase in neutrophil surface density of CD11b/CD18 is a promising and a highly effective sepsis marker for diagnosing early-onset infection in term neonates as well. $(33,42)$ The results of those studies show, on the first day of life, a two- to fourfold increase in neutrophil CD11b expression in infants with blood-culture positive sepsis. This increase is similar to that demonstrated in neutrophils from adults with bloodculture positive sepsis. (31) Similarly, in 2012, Genel et al. (43) reported that neutrophil and monocyte CD11b expressions were significantly elevated in infected neonates as compared with the control group of subjects. They also reported that neutrophil CD11b had a sensitivity of $66 \%$ and specificity of $71 \%$ for detecting neonatal infection, while monocyte CD11b had a sensitivity of $70 \%$ and specificity of $62 \%$ for detecting neonatal infection. (43) However, decreased values of the CD11b expression on neutrophils in sepsis were also found. (44)

During rolling, the adhesive contact zone between the cell and the vessel is rapidly translated along the vessel wall. This requires the rapid breakage and formation of adhesive bonds. As mentioned earlier, only certain adhesion molecules, including selectins and some integrins, have been found to support rolling. Chen et al. (45) have identified antibodies, all of the IgM class, to carbohydrate antigens CD15 and CD15s. Lewis $\mathbf{x}$ (CD15) and sialyl-Lewis $\mathbf{x}$ (CD15s) are expressed on circulating neutrophils and monocytes and, upon recognition by endothelial selectins, mediate leukocyte endothelium interactions and mediate tethering and rolling by serving as the ligands for E and P-selectin. CD15 has an intracellular pool in the membranes of the primary and secondary granules, and surface expression is increased upon stimulation with 
chemotactic peptides. Zen et al., in 2007, have shown that both CD11b and CD18 subunits of Mac-1 purified from normal human neutrophils are decorated with $\mathrm{sLe}^{\mathrm{x}}$ or $\mathrm{sLe}^{\mathrm{x}}$ related moieties that mediate the binding of $\beta 2$ integrin with E-selectin. (46) The ligation of $\mathrm{sLe}^{\mathrm{x}}$ moieties by specific anti-sLe ${ }^{\mathrm{x}}$ antibody significantly affects neutrophil functions including induction of neutrophil aggregation, induction of secondary granule release and impairment of neutrophil transmigration. The authors also observed that $\mathrm{N}$-formyl-methionine-leucine-phenylalanine (fMLP) stimulation strongly enhanced the neutrophil cell surface expression of $\mathrm{sLe}^{\mathrm{X}}$ moieties and the up-regulation pattern was similar to that of Mac-1 with fMLP stimulation. (46) So far, only one research evaluated the CD15s as predictor of SBI and its role in differentiation between bacterial and viral infection. (47) It was found that the percentage of CD15s+ neutrophils (\%CD15s+) and absolute count of CD15s+ neutrophils (ACCD15s+) were significantly different between the groups and both of them proved to be valuable biomarkers for differentiation between bacterial and viral infection.

The surface receptors of neutrophils recognize bacterial antigens and this interaction activates the neutrophils to phagocyte. Neutrophil binding of bacteria is greatly augmented when the pathogens are coated with IgG. $\mathrm{Fc} \gamma$ receptor I ( $\mathrm{Fc} \gamma \mathrm{RI}$ ), which is the high affinity receptor for monomeric IgG1 and $\mathrm{IgG}_{3}$, is recognized by the monoclonal antibody CD64. (48) It has a molecular weight of $72 \mathrm{kDa}$ and it encoded by three genes on the long arm of chromosome 1. CD64 is expressed only to a very low extent on resting neutrophils, (12) and is considered to be a marker of activated neutrophils. The quantification of CD64 expression on the surface of neutrophils by flow cytometry has been proposed as a diagnostic test for sepsis in neonates, $(30,49,50)$ and hospitalized children. $(51,52)$ In neonatology, CD64 has been used to identify premature and term neonates with sepsis. In most of these studies, CD64 has appeared to perform moderately well, often in combination with other markers or hematologic parameters. Ng et al. showed that CD64 levels had high sensitivity (95-97\%) and negative predictive value (97-99\%) for earlyand late-onset infection in infants of very low birth weight. $(37,49)$ Using the additional biomarkers like IL- 6 or CRP, the sensitivity was increased 
to $100 \%$ and the specificity exceeding $88 \%$ was noticed. (37) LaysecaEspinosa et al. also found that expression of CD64 is a highly specific laboratory indicator of neonatal sepsis (96.8\%), although with a low sensitivity (25.8\%). (30) Their data indicate that the high specificity of elevated levels of CD64 for neonatal sepsis is achieved through a single determination and the high percentage of CD64+ cells seen in neonatal sepsis is maintained in these patients for at least $24 \mathrm{~h}$. A large cohort study also demonstrated that neutrophil CD64 was substantially upregulated and useful (sensitivity $96 \%$ and specificity $81 \%$ ) for identifying neonates with early-onset clinical sepsis and pneumonia. (49) The authors reported that additional use of CRP did not significantly improve the sensitivity of neutrophil CD64, but adversely affected the specificity of the test. In critically ill neonates and children, it was found that diagnostic accuracy of CD64 index was higher than the diagnostic accuracy of routine laboratory markers PCT and CRP at the time of suspected sepsis and $24 \mathrm{~h}$ later. (51) A similar conclusion was reported by Davis et al. (53) In their research, CD64 had high diagnostic performance as a predictor of sepsis (sensitivity $87.9 \%$, specificity $71.2 \%$ ), and was found to be a better diagnostic biomarker of infection/sepsis when compared with CRP, band counts, and absolute neutrophil counts. (53)

Cid et al. (54) recently conducted a meta-analysis of the literature on CD64 and calculated mean pooled results for various performance characteristics. Overall, for adult and pediatric patients, pooled sensitivity was $79 \%$ and specificity $91 \%$ for neutrophil CD64 expression as marker of bacterial infection. For the pediatric studies, they found a mean sensitivity of $71 \%$ and a mean specificity of $87 \%$ for CD64. (54)

\section{Other biomarkers}

A number of acute phase proteins including alpha1-antitrypsin, fibronectin, haptoglobin, lactoferrin, neopterin, inter-a inhibitor proteins, granulocyte colony stimulating factor, orosomucoid, and antithrombin have been evaluated in relation to neonatal sepsis. Although these acute phase proteins may be candidate biomarkers for sepsis, none has been routinely used clinically or studied on a large scale.

Serum amyloid A (SAA) is an acute phase protein induced by the 
proinflammatory cytokines. There is a significant increase in SAA levels from 8 to $24 \mathrm{~h}$ after the onset of sepsis and it was shown that SAA had better diagnostic accuracy than CRP at septic evaluation in neonatal early onset sepsis. (55) Interleukin-12 was measured in newborns at the time when sepsis was first suspected clinically and was higher in patients with sepsis than in those without. (56) Interferon-induced protein 10 (IP-10) was higher in neonates with sepsis and necrotizing enterocolitis than in neonates who had only necrotizing enterocolitis. (57)

Lipopolysaccharide-binding protein (LBP), a 50-kDa acute-phase protein, is mainly synthesized in the liver. It binds with high affinity to lipopolysaccharide (LPS) in the plasma, transfers LPS to membrane bound or soluble CD14, and modulates the microbial-induced activation of the inflammatory host response. It was reported that LBP has better sensitivity and specificity for detecting sepsis than LPS-soluble, CD14 complexes, and PCT in early onset sepsis, but is equally effective as CRP in detecting sepsis in neonates older than $48 \mathrm{~h}$. (58)

\section{Conclusion}

In conclusion, it is evident that, to date, none of the above mentioned biomarkers alone or in combination are $100 \%$ sensitive and specific, allowing neonatologists or pediatricians to withhold antibiotic treatment in an infant or older child with clinical signs suggestive of infection. Furthermore, analysis of some biomarkers in the clinical setting, like cell surface markers, requires specialized equipment and qualified personnel readily available. This limits the practical application of this technology. Therefore, the search for better biomarkers continues...

\section{References}

1. Levine D, Platt S, Dayan P, Macias C, Zorc J, Krief W, et al. Risk of serious bacterial infection in young febrile infants with respiratory syncytial virus infections. Pediatrics 2004;113(6):1728-34.

2. Ashkenazi-Hoffnung L, Livni G, Amir J, Bilavsky E. Serious bacterial infections in hospitalized febrile infants aged 90 days or younger: The traditional combination of ampicillin and gentamicin is still appropriate. 
Scandin J Infect Dis 2011;43(6-7):489-94.

3. Bilavsky E, Yarden-Bilavsky H, Ashkenazi S, Amir J. C-reactive protein as a marker of serious bacterial infections in hospitalized febrile infants. Acta Paediatrica 2009;98(11):1776-80.

4. Levy M, Fink M, Marshall J, Abraham E, Angus D, Cook D, et al. 2001 SCCM/ESICM/ACCP/ATS/SIS International Sepsis Definitions Conference. Crit Care Med 2003;31(4):1250-6.

5. Angus D, Linde-Zwirble W, Lidicker J, Clermont G, Carcillo J, Pinsky M. Epidemiology of severe sepsis in the United States: Analysis of incidence, outcome, and associated costs of care. Crit Care Med 2001;29(7):1303-10.

6. Olaciregui I, Hernandez U, Munoz J, Emparanza J, Landa J. Markers that predict serious bacterial infection in infants under 3 months of age presenting with fever of unknown origin. Arch Dis Childhood 2009;94(7):501-5.

7. Dellinger R, Levy M, Carlet J, Bion J, Parker M, Jaeschke R, et al. Surviving Sepsis Campaign: International guidelines for management of severe sepsis and septic shock: 2008. Crit Care Med 2008;36(1):296-327.

8. Ng P, Lam H. Diagnostic markers for neonatal sepsis. Curr Opin Ped 2006;18(2):125-31.

9. Marshall J, Reinhart K, Forum IS. Biomarkers of sepsis. Crit Care Med 2009;37(7):2290-8.

10. Atkinson A, Colburn W, DeGruttola V, DeMets D, Downing G, Hoth D, et al. Biomarkers and surrogate endpoints: Preferred definitions and conceptual framework. Clin Pharm Therap 2001;69(3):89-95.

11. Kaplan J, Wong H. Biomarker discovery and development in pediatric critical care medicine. Ped Crit Care Med 2011;12(2):165-73.

12. Davis B. Improved diagnostic approaches to infection/sepsis detection. Exp Rev Mol Diag 2005;5(2):193-207.

13. Zeitoun A, Gad S, Attia F, Abu Maziad A, Bell E. Evaluation of neutrophilic CD64, interleukin 10 and procalcitonin as diagnostic markers of early- and late-onset neonatal sepsis. Scandin J Infect Dis 2010;42(4):299-305.

14. Lacour A, Gervaix A, Zamora S, Vadas L, Lombard P, Dayer J, et al. Procalcitonin, IL-6, IL-8, IL-1 receptor antagonist and C-reactive protein as identificators of serious bacterial infections in children with fever without localising signs. Europ J Ped 2001;160(2):95-100. 
15. Bonsu B, Harper M. Identifying febrile young infants with bacteremia: Is the peripheral white blood cell count an accurate screen? Ann Emerg Med 2003;42(2):216-25.

16. Galetto-Lacour A, Zamora S, Gervaix A. Bedside procalcitonin and Creactive protein tests in children with fever without localizing signs of infection seen in a referral center. Pediatrics 2003;112(5):1054-60.

17. Rudinsky S, Carstairs K, Reardon J, Simon L, Riffenburgh R, Tanen D. Serious Bacterial Infections in Febrile Infants in the Post-Pneumococcal Conjugate Vaccine Era. Acad Emerg Med 2009;16(7):585-90.

18. Du Clos T. Function of C-reactive protein. Ann Med 2000;32(4):274-8.

19. Couto R, Barbosa J, Pedrosa T, Biscione F. C-reactive protein-guided approach may shorten length of antimicrobial treatment of cultureproven late-onset sepsis. An intervention study. Brazil J Infect Dis 2007;11(2):240-5.

20. Franz A, Steinbach G, Kron M, Pohlandt F. Reduction of unnecessary antibiotic therapy in newborn infants using interleukin-8 and C-reactive protein as markers of bacterial infections. Pediatrics 1999;104(3):447-53.

21. Pulliam PN, Attia MW, Cronan KM. C-reactive protein in febrile children 1 to 36 months of age with clinically undetectable serious bacterial infection. Pediatrics 2001;108(6):1275-9.

22. Tang B, Eslick G, Craig J, McLean A. Accuracy of procalcitonin for sepsis diagnosis in critically ill patients: systematic review and meta-analysis. Lancet Infect Dis 2007;7(3):210-7.

23. Lopez A, Cubells C, Garcia J, Pou J, Emergencies SSP. Procalcitonin in pediatric emergency departments for the early diagnosis of invasive bacterial infections in febrile infants: results of a multicenter study and utility of a rapid qualitative test for this marker. Ped Infect Dis J 2003;22(10):895-903.

24. Prat C, Dominguez J, Rodrigo C, Gimenez M, Azuara M, Blanco S, et al. Use of quantitative and semiquantitative procalcitonin measurements to identify children with sepsis and meningitis. Europ J Clin Microb Infect Dis 2004;23(2):136-8.

25. Carrol E, Newland P, Riordan F, Thomson A, Curtis N, Hart C. Procalcitonin as a diagnostic marker of meningococcal disease in children presenting with fever and a rash. Arch Dis Childhood 2002;86(4):282-5.

26. Gendrel D, Raymond J, Coste J, Moulin F, Lorrot M, Guerin S, et al. 
Comparison of procalcitonin with C-reactive protein, interleukin 6 and interferon-alpha for differentiation of bacterial vs. viral infections. Ped Infect Dis J 1999;18(10):875-81.

27. Berner R, Niemeyer C, Leititis J, Funke A, Schwab C, Rau U, et al. Plasma levels and gene expression of granulocyte colony-stimulating factor, tumor necrosis factor-alpha, interleukin (IL)-1 beta, IL-6, IL-8, and soluble intercellular adhesion molecule- 1 in neonatal early onset sepsis. Ped Research 1998;44(4):469-77.

28. Sullivan J, kilpatrick L, Costarino A, Lee S, Harris M. Correlation of plasma cytokine elevations with mortality-rate in children with sepsis. $\mathrm{J}$ Ped 1992;120(4):510-5.

29. Strait R, Kelly K, Kurup V. Tumor necrosis factor-alpha, interleukin-1 beta, and interleukin-6 levels in febrile, young children with and without occult bacteremia. Pediatrics 1999;104(6):1321-6.

30. Layseca-Espinosa E, Perez-Gonzalez L, Torres-Montes A, Baranda L, de la Fuente H, Rosenstein Y, et al. Expression of CD64 as a potential marker of neonatal sepsis. Ped Allerg Immunol 2002;13(5):319-27.

31. 31. Takala A, Jousela I, Jansson S, Olkkola K, Takkunen O, Orpana A, et al. Markers of systemic inflammation predicting organ failure in community-acquired septic shock. Clin Sci 1999;97(5):529-38.

32. 32. Franz A, Kron M, Pohlandt F, Steinbach G. Comparison of procalcitonin with interleukin 8 , C-reactive protein and differential white blood cell count for the early diagnosis of bacterial infections in newborn infants. Ped Infect Dis J 1999;18(8):666-71.

33. 33. Nupponen I, Andersson S, Järvenpää AL, Kautiainen H, Repo H. Neutrophil CD11b expression and circulating interleukin-8 as diagnostic markers for early-onset neonatal sepsis. Pediatrics 2001;108(1):E12.

34. 34. Cusumano V, Midiri A, Cusumano V, Bellantoni A, De Sossi G, Teti G, et al. Interleukin-18 is an essential element in host resistance to experimental group B streptococcal disease in neonates. Infect Immunol 2004;72(1):295-300.

35. 35. Kingsmore S, Kennedy N, Halliday H, Van Velkinburgh J, Zhong S, Gabriel V, et al. Identification of Diagnostic Biomarkers for Infection in Premature Neonates. Molec Cell Proteom 2008;7(10):1863-75.

36. 36. Bender L, Thaarup J, Varming K, Krarup H, Ellermann-Eriksen S, Ebbesen F. Early and late markers for the detection of early-onset 
neonatal sepsis. Danish Med Bull 2008;55(4):219-23.

37. 37. Ng P, Cheng S, Chui K, Fok T, Wong M, Wong W, et al. Diagnosis of late onset neonatal sepsis with cytokines, adhesion molecule, and Creactive protein in preterm very low birthweight infants. Arch Dis Childhood 1997;77(3):F221-F7.

38. 38. Gonzalez-Amaro R, Sanchez-Madrid F. Cell adhesion molecules: Selectins and integrins. Crit Rev Immunol 1999;19(5-6):389-429.

39. 39. Sengelov H, Kjeldsen L, Diamond M, Springer T, Borregaard N. Subcellular-localization and dynamics of mac-1 (alpha(m)beta(2)) in human neutrophils. J Clin Invest 1993;92(3):1467-76.

40. 40. Kobold A, Tulleken J, Zijlstra J, Sluiter W, Hermans J, Kallenberg C, et al. Leukocyte activation in sepsis; correlations with disease state and mortality. Int Care Med 2000;26(7):883-92.

41. 41. Turunen R, Andersson S, Nupponen I, Kautiainen H, Siitonen S, Repo $\mathrm{H}$. Increased CD11b-density on circulating phagocytes as an early sign of late-onset sepsis in extremely low-birth-weight infants. Ped Research 2005;57(2):270-5.

42. 42. Weirich E, Rabin R, Maldonado Y, Benitz W, Modler S, Herzenberg L. Neutrophil CD11b expression as a diagnostic marker for early-onset neonatal infection. J Ped 1998;132(3):445-51.

43. 43. Genel F, Atlihan F, Gulez N, Kazanci E, Vergin C, Terek D, et al. Evaluation of adhesion molecules CD64, CD11b and CD62L in neutrophils and monocytes of peripheral blood for early diagnosis of neonatal infection. World J Ped 2012;8(1):72-5.

44. 44. Nakae H, Endo S, Inada K, Takakuwa T, Kasai T. Changes in adhesion molecule levels in sepsis. Research Communications in Molecular Pathol Pharm 1996;91(3):329-38.

45. 45. Chen S, Alon R, Fuhlbrigge R, Springer T. Rolling and transient tethering of leukocytes on antibodies reveal specializations of selectins. Proceedings of the National Academy of Sciences of the United States of America. 1997;94(7):3172-7.

46. 46. Zen K, Cui L, Zhang C, Liu Y. Critical role of Mac-1 sialyl Lewis X moieties in regulating neutrophil degranulation and transmigration. $\mathrm{J}$ Mol Biol 2007;374(1):54-63.

47. 47. Markic J, Jeroncic A, Polancec D, Bosnjak N, Markotic A, Mestrovic J, et al. CD15s is a potential biomarker of serious bacterial infection in 
infants admitted to hospital. Eur J Pediatr 2013;172(10):1363-9.

48. 48. Hoffmann J. Neutrophil CD64: a diagnostic marker for infection and sepsis. Clin Chem Lab Med 2009;47(8):903-16.

49. 49. Ng P, Li G, Chui K, Chu W, Li K, Wong R, et al. Neutrophil CD64 is a sensitive diagnostic marker for early-onset neonatal infection. Ped Research 2004;56(5):796-803.

50. 50. Bhandari V, Wang C, Rinder C, Rinder H. Hematologic profile of sepsis in neonates: Neutrophil CD64 as a diagnostic marker. Pediatrics 2008;121(1):129-34.

51. 51. Groselj-Grenc M, Ihan A, Pavcnik-Arnol M, Kopitar A, GmeinerStopar T, Derganc M. Neutrophil and monocyte CD64 indexes, lipopolysaccharide-binding protein, procalcitonin and C-reactive protein in sepsis of critically ill neonates and children. Int Care Med 2009;35(11):1950-8.

52. 52. Fjaertoft G, Hakansson L, Pauksens K, Sisask G, Venge P. Neutrophil CD64 (Fc gamma RI) expression is a specific marker of bacterial infection: A study on the kinetics and the impact of major surgery. Scand J Infect Dis 2007;39(6-7):525-35.

53. 53. Davis B, Olsen S, Ahmad E, Bigelow N. Neutrophil CD64 is an improved indicator of infection or sepsis in emergency department patients. Arch Pathol Lab Med 2006;130(5):654-61.

54. 54. Cid J, Aguinaco R, Sanchez R, Garcia-Pardo G, Llorente A. Neutrophil CD64 expression as marker of bacterial infection: A systematic review and meta-analysis. J Infect 2010;60(5):313-9.

55. 55. Arnon S, Litmanovitz I, Regev R, Bauer S, Shainkin-Kestenbaum R, Dolfin T. Serum amyloid A: an early and accurate marker of neonatal early-onset sepsis. J Perinat 2007;27(5):297-302.

56. 56. Sherwin C, Broadbent R, Young S, Worth J, McCaffrey F, Medlicott N, et al. Utility of Interleukin-12 and Interleukin-10 in Comparison with Other Cytokines and Acute-Phase Reactants in the Diagnosis of Neonatal Sepsis. Am J Perin 2008;25(10):629-36.

57. 57. Ng P, Li K, Chui K, Leung T, Wong R, Chu W, et al. IP-10 is an early diagnostic marker for identification of late-onset bacterial infection in preterm infants. Ped Research 2007;61(1):93-8.

58. 58. Pavcnik-Arnol M, Hojker S, Derganc M. Lipopolysaccharide-binding protein, lipopolysaccharide, and soluble CD14 in sepsis of critically ill 
neonates and children. Int Care Med 2007;33(6):1025-32.

Josko Markic

Department of Pediatrics

University Hospital Centre Split

Spinciceva 1, 21000 Split, Croatia

Phone: +385-21-556-686

Fax: +385-21-556-590

E-mail: jmarkic@mefst.hr

Article printed from Signa Vitae: http://www.signavitae.com

URL to article: http://www.signavitae.com/2015/12/biomarkersof-sepsis-in-neonates-and-children-review-article/

Copyright (C) 2015 Signa Vitae. All rights reserved. 